\title{
ON NONOPTIMALITY OF BOLD PLAY FOR SUBFAIR RED-AND-BLACK WITH A RATIONAL-VALUED HOUSE LIMIT
}

\author{
MAY-RU CHEN, ${ }^{*}$ Academia Sinica \\ PEI-SHOU CHUNG, ${ }^{* *}$ National Hsinchu Girls' Senior High School \\ SHOOU-REN HSIAU, ${ }^{* * *}$ National Changhua University of Education \\ YI-CHING YAO, ${ }^{* * * *}$ Academia Sinica and National Chengchi University
}

\begin{abstract}
In the subfair red-and-black gambling problem, a gambler can stake any amount in his possession, winning an amount equal to the stake with probability $w$ and losing the stake with probability $1-w$, where $0<w<\frac{1}{2}$. The gambler seeks to maximize the probability of reaching a fixed fortune (to be normalized to unity) by gambling repeatedly with suitably chosen stakes. In their classic work, Dubins and Savage (1965), (1976) showed that it is optimal to play boldly. When there is a house limit of $\ell\left(0<\ell<\frac{1}{2}\right)$, so that the gambler can stake no more than $\ell$, Wilkins (1972) showed that bold play remains optimal provided that $1 / \ell$ is an integer. On the other hand, building on an earlier surprising result of Heath, Pruitt and Sudderth (1972), Schweinsberg (2005) recently showed that, for all irrational $0<\ell<\frac{1}{2}$ and all $0<w<\frac{1}{2}$, bold play is not optimal for some initial fortune. The purpose of the present paper is to present several results supporting the conjecture that, for all rational $\ell$ with $1 / \ell$ not an integer and all $0<w<\frac{1}{2}$, bold play is not optimal for some initial fortune. While most of these results are based on Schweinsberg's method, in a special case where his method is shown to be inapplicable, we argue that the conjecture can be verified with the help of symbolic-computation software.
\end{abstract}

Keywords: Gambling theory; red-and-black; bold play; optimal strategy

2000 Mathematics Subject Classification: Primary 60G40

Secondary 91A60

\section{Introduction}

In the subfair red-and-black gambling problem, a gambler can stake any amount $s$ of his fortune $f, 0 \leq s \leq f<1$. If he stakes an amount $s$, his fortune becomes $f+s$ with probability $w$ and $f-s$ with probability $1-w$, where the win probability $w$ satisfies $0<w<\frac{1}{2}$. The gambler seeks to maximize the probability of reaching a fortune of 1 (the goal) by gambling repeatedly with suitably chosen stakes. Dubins and Savage [2], [3] showed that it is optimal to play boldly, i.e. to stake $\min \{f, 1-f\}$ if the current fortune is $f, 0<f<1$. (In words, to

Received 3 June 2008; revision received 5 September 2008.

* Current address: Department of Applied Mathematics, National Sun Yat-sen University, 70 Lien-hai Road, Kaohsiung 804, Taiwan, R.O.C. Email address: chenmr@math.nsysu.edu.tw

** Postal address: c/o Prof. S.-R. Hsiau, Department of Mathematics, National Changhua University of Education, Jin-De Campus, Chang-Hua 500, Taiwan, R.O.C. Email address: igo.tw@ yahoo.com.tw

*** Postal address: Department of Mathematics, National Changhua University of Education, Jin-De Campus, ChangHua 500, Taiwan, R.O.C. Email address: srhsiau@cc.ncue.edu.tw

**** Postal address: Institute of Statistical Science, Academia Sinica, Taipei 115, Taiwan, R.O.C.

Email address: yao@stat.sinica.edu.tw 
play boldly is to stake on each play as much as possible without risk of overshooting the goal.) The Dubins-Savage optimality result on the bold strategy has been extended in several ways; see [7] for a brief review.

One of the extensions is subfair red-and-black with a house limit of $\ell$ in which the gambler can stake no more than $\ell$. Under the house limit constraint, to play boldly is to stake $\min \{f, 1-f, \ell\}$ if the current fortune is $f, 0<f<1$. Since $\min \{f, 1-f\}=\min \{f, 1-f, \ell\}$ for $\ell \geq \frac{1}{2}$, the bold strategy is optimal if $\ell \geq \frac{1}{2}$. So we assume that $0<\ell<\frac{1}{2}$ hereafter. Wilkins [6] proved that it is optimal to play boldly if $\ell=1 / j$ for some integer $j \geq 3$. Heath et al. [4] showed, however, that if $\ell$ either satisfies $1 /(j+1)<\ell<1 / j$ for some integer $j \geq 3$ or is irrational with $\frac{1}{3}<\ell<\frac{1}{2}$, then there exists an $\varepsilon>0$ such that, for all $0<w<\varepsilon$, the bold strategy is not optimal for some initial fortune $f$. Their result has been improved by Schweinsberg [5], who showed that, for all irrational $0<\ell<\frac{1}{2}$ and all $0<w<\frac{1}{2}$, the bold strategy is not optimal for some initial fortune $f$. While Schweinsberg's result settles the case of irrational $\ell$, the following conjecture remains open.

Conjecture 1. For all rational $0<\ell<\frac{1}{2}$ with $1 / \ell$ not an integer and all $0<w<\frac{1}{2}$, the bold strategy is not optimal for some initial fortune $f$.

Let $U_{\ell, w}(f)$ denote the maximum probability of reaching the goal with initial fortune $f$, which depends on $\ell$ (house limit) and $w$ (win probability of each bet). Let $Q_{\ell, w}(f)$ denote the probability that the gambler with initial fortune $f$ reaches the goal under the bold strategy. Then Conjecture 1 states that, for all rational $0<\ell<\frac{1}{2}$ with $1 / \ell$ not an integer,

$$
\left\{f \in(0,1): Q_{\ell, w}(f)<U_{\ell, w}(f)\right\} \neq \varnothing \quad \text { for all } 0<w<\frac{1}{2} .
$$

The purpose of the present paper is to present partial results in support of Conjecture 1. As these results make extensive use of the tools developed by Schweinsberg [5], his method is briefly reviewed in Section 2 . Note that a rational $\ell$ with $0<\ell<\frac{1}{2}$ and $1 / \ell \notin\{3,4, \ldots\}$ has a unique representation of the form

$$
\ell=\frac{n}{q n+r} \quad \text { with integers } n>r \geq 1, q \geq 2, n \text { and } r \text { coprime. }
$$

In Section 3 we show that (1) holds provided that the integer $r$ in (2) is even. In Section 4 we consider the case in which $\ell=n /(2 n+1)$ for $n \geq 2$ (i.e. $q=2$ and $r=1$ in (2)). In addition to its mathematical tractability, the essential reason for considering this special case is that if (1) does not hold for some values of $\ell$ then intuitively such values are likely to be close to $\frac{1}{2}$. In other words, if Conjecture 1 is false, we would expect to find counterexamples with $\ell$ close to $\frac{1}{2}$. By making use of simple number-theoretic results we prove that (1) holds if $\ell=n /(2 n+1)$ satisfies the following condition.

There exists a positive integer $k<2 n$ such that $2^{k} \equiv 1(\bmod 2 n+1)$.

Since 2 and $2 n+1$ are coprime, we have $2^{\phi(2 n+1)} \equiv 1(\bmod 2 n+1)$ by Euler's theorem, where $\phi(m)$, Euler's totient function, is the number of positive integers less than or equal to $m$ that are coprime to $m$; see [1, p. 9]. Clearly, $\phi(m) \leq m-1$ for $m \geq 2$ and $\phi(m)<m-1$ if $m>1$ is not a prime. So (3) is satisfied if $2 n+1$ is not a prime. On the other hand, we show in Section 5 that Schweinsberg's method cannot be used to prove (1) when condition (3) is not satisfied, in which case it is argued that (1) may still be established by using symbolic-computation software. 


\section{Schweinsberg's method}

Denote by $X_{i}=X_{i}^{\ell, w}$ the gambler's fortune after $i$ plays under the bold strategy. Then $\left\{X_{i}: i=0,1, \ldots\right\}$ is a Markov chain with transition probabilities given by

$$
\mathrm{P}\left(X_{i+1}=f+b(f) \mid X_{i}=f\right)=w, \quad \mathrm{P}\left(X_{i+1}=f-b(f) \mid X_{i}=f\right)=1-w,
$$

where $0 \leq f \leq 1$ and the function $b:[0,1] \rightarrow[0,1]$ is defined by $b(f)=\min \{f, 1-f, \ell\}$ (the bold stake for the gambler when his fortune is $f$ ). Note that the transition probabilities depend on $w$ and $\ell$, that the Markov chain has two absorbing states 0 and 1, and that

$$
Q_{\ell, w}(f)=\mathrm{P}\left(X_{i}=1 \text { for some } i \geq 0 \mid X_{0}=f\right) .
$$

Define

$$
S_{\ell}=\left\{f \in(0,1): \mathrm{P}\left(X_{i}=1-\ell \text { for some } i \geq 0 \mid X_{0}=f\right)>0\right\},
$$

the set of all $f$ such that the gambler, with initial fortune $f$ and playing boldly, could have a fortune of $1-\ell$ in a finite number of plays. Note that $1-\ell \in S_{\ell}$ and that, for $0<\ell<\frac{1}{2}$ and $0<w<\frac{1}{2}$, the set $S_{\ell}$ depends on $\ell$ but not on $w$.

Schweinsberg's method consists of the following two key results.

(i) For given $0<\ell<\frac{1}{2}, 0<w<\frac{1}{2}$, and $f \in S_{\ell}$, there exists a constant $C=C(\ell, w, f)>$ 0 such that $Q_{\ell, w}(f)-Q_{\ell, w}(f-\varepsilon) \geq C(1-w)^{-\log \varepsilon}$ whenever $0<\varepsilon<\min \{\ell, f\}$.

(ii) For given $0<\ell<\frac{1}{2}, 0<w<\frac{1}{2}$, and $f \in(0,1) \backslash S_{\ell}$, for every $C>0$, there exists a $\delta>0$ such that $Q_{\ell, w}(f)-Q_{\ell, w}(f-\varepsilon) \leq C(1-w)^{-\log \varepsilon}$ whenever $0<\varepsilon<\delta$.

For a given $0<\ell<\frac{1}{2}$, suppose that there exists an $f \in(\ell, 1-\ell)$ such that $f-\ell \in S_{\ell}$ and $f+\ell \notin S_{\ell}$. Then, by (i) and (ii), for every $0<w<\frac{1}{2}$, we have

$$
\begin{aligned}
Q_{\ell, w}(f-\varepsilon) & =w Q_{\ell, w}(f+\ell-\varepsilon)+(1-w) Q_{\ell, w}(f-\ell-\varepsilon) \\
& <w Q_{\ell, w}(f+\ell-2 \varepsilon)+(1-w) Q_{\ell, w}(f-\ell) \\
& \leq U_{\ell, w}(f-\varepsilon)
\end{aligned}
$$

for sufficiently small $\varepsilon>0$, from which the lemma below follows.

Lemma 1. ([5].) For a given $0<\ell<\frac{1}{2}$, if there exists an $f \in(\ell, 1-\ell)$ such that $f-\ell \in S_{\ell}$ and $f+\ell \notin S_{\ell}$, then (1) holds.

\section{The case in which $\ell=n /(q n+r)$ with $r$ even}

In this section we assume that $r$ is even in (2). Writing $r=2 t$, we have

$$
\ell=\frac{n}{q n+2 t}, \quad q \geq 2,2 \leq 2 t<n, n \text { and } 2 t \text { coprime. }
$$

Note that (6) implies that $n$ is odd. Define two functions $T_{+, \ell}$ and $T_{-, \ell}:[0,1] \rightarrow[0,1]$ by

$$
\begin{aligned}
& T_{+, \ell}(f)= \begin{cases}2 f & \text { if } 0 \leq f \leq \ell, \\
f+\ell & \text { if } \ell<f<1-\ell, \\
2 f-1 & \text { if } 1-\ell \leq f \leq 1,\end{cases} \\
& T_{-, \ell}(f)= \begin{cases}2 f & \text { if } 0 \leq f \leq \ell, \\
f-\ell & \text { if } \ell<f<1-\ell, \\
2 f-1 & \text { if } 1-\ell \leq f \leq 1 .\end{cases}
\end{aligned}
$$


Note that $T_{+, \ell}(f)=T_{-, \ell}(f)$ for $f \in[0, \ell] \cup[1-\ell, 1]$ and that

$$
\mathrm{P}\left(X_{i+1} \in\left\{T_{+, \ell}(f), T_{-, \ell}(f), 0,1\right\} \mid X_{i}=f\right)=1 \text { for } 0<f<1,
$$

where $\left\{X_{i}\right\}$ is the Markov chain defined in (4).

Let

$$
A_{\ell}=\left\{\frac{j}{q n+2 t}: j=1,2, \ldots, q n+2 t-1\right\} .
$$

For notational simplicity, we will drop the subscript $\ell$ in $A_{\ell}, S_{\ell}, T_{+, \ell}$, and $T_{-, \ell}$ unless we need to emphasize their dependence on $\ell$.

Lemma 2. Assume that $\ell$ satisfies (6). Then

$$
A \cap S\left(=A_{\ell} \cap S_{\ell}\right)=\{1-j \ell: j=1, \ldots, q-1\} .
$$

Proof. We first show that $B:=\{1-j \ell: j=1, \ldots, q-1\} \subset A \cap S$. Note that $B \subset A$, that $1-\ell \in A \cap S$, and that, for $1<j \leq q-1$,

$$
\ell=\frac{n}{q n+2 t}<\frac{(q-j) n+2 t}{q n+2 t}=1-j \ell<1-\ell .
$$

So, for $1<j \leq q-1$,

$$
T_{+}^{j-1}(1-j \ell)=T_{+}^{j-2}\left(T_{+}(1-j \ell)\right)=T_{+}^{j-2}(1-(j-1) \ell)=\cdots=1-\ell,
$$

implying that, by the definition of $S,(5), 1-j \ell \in S$. This proves that $B \subset A \cap S$.

To prove $A \cap S \subset B$, we claim that

$$
T_{+}(x), T_{-}(x) \in A \backslash B \quad \text { for all } x \in A \backslash B,
$$

which together with (9) implies that, if $X_{0} \in A \backslash B$, the Markov chain $\left\{X_{i}\right\}$ can never leave $A \backslash B$ before it enters an absorbing state of $\{0,1\}$. In particular, the Markov chain can never visit the state $1-\ell$ if it starts in $A \backslash B$. This shows that, by the definition of $S$, (5), no element of $A \backslash B$ is in $S$, so $A \cap S \subset B$.

It remains to prove (11). Let

$$
x=\frac{k}{q n+2 t} \in A \backslash B \quad \text { with } k \neq(q-j) n+2 t, j=1, \ldots, q-1 .
$$

It suffices to show that $T_{+}(x) \notin B$ and $T_{-}(x) \notin B$ for each of the three cases $0<x \leq \ell$, $\ell<x<1-\ell$, and $1-\ell<x<1$. (Note that $x \neq 1-\ell \in B$.)

If $0<x \leq \ell$ (i.e. $0<k \leq n)$ then $T_{+}(x)=T_{-}(x)=2 x=2 k /(q n+2 t)$. Since $n$ is odd by (6), we have $2 k \neq n+2 t=(q-(q-1)) n+2 t$. Also, $k \leq n$ implies that $2 k<(q-j) n+2 t$ for $j=1, \ldots, q-2$. So $T_{+}(x)=T_{-}(x)=2 k /(q n+2 t) \notin B=$ $\{((q-j) n+2 t) /(q n+2 t): j=1, \ldots, q-1\}$.

If $1-\ell<x<1$ then $(q-1) n+2 t<k<q n+2 t$, or, equivalently,

$$
(q-2) n+2 t<2 k-(q n+2 t)<q n+2 t .
$$

Also, $T_{+}(x)=T_{-}(x)=2 x-1=(2 k-(q n+2 t)) /(q n+2 t)$. Since $n$ is odd, we have $2 k-(q n+2 t) \neq(q-1) n+2 t$, which together with (13) implies that $2 k-(q n+2 t) \neq$ $(q-j) n+2 t, j=1, \ldots, q-1$. So $T_{+}(x)=T_{-}(x)=(2 k-(q n+2 t)) /(q n+2 t) \notin B$. 
If $\ell<x<1-\ell$ then $n<k<(q-1) n+2 t$, which together with $k \neq(q-j) n+2 t, j=$ $1, \ldots, q-1$ (by (12)), implies that $n \nmid(k-2 t)$. So $n \nmid(k+n)-2 t$ and $n \nmid(k-n)-2 t$, implying that $k \pm n \neq(q-j) n+2 t, j=1, \ldots, q-1$. It follows that

$$
T_{+}(x)=x+\ell=\frac{k+n}{q n+2 t} \notin B, \quad T_{-}(x)=x-\ell=\frac{k-n}{q n+2 t} \notin B .
$$

The proof is complete.

Theorem 1. Assume that $\ell$ satisfies (6). Then there exists an $f \in(\ell, 1-\ell)$ such that $f-\ell \in S$ and $f+\ell \notin S$.

Theorem 1 follows from Lemmas 3, 4, and 5, below. By Theorem 1 and Lemma 1, (1) holds for $\ell$ satisfying (6).

Lemma 3. Assume that $\ell=n /(q n+2 t)$ satisfies (6) with $q \geq 3$ (i.e. $\left.\ell<\frac{1}{3}\right)$. Let $\lambda=$ $(1+(q-5) \ell) / 2$. Then

(i) $0<\lambda<1-2 \ell$;

(ii) $\lambda \in S$;

(iii) $\lambda+2 \ell \notin S$.

Consequently, we have $\lambda+\ell \in(\ell, 1-\ell),(\lambda+\ell)-\ell \in S$, and $(\lambda+\ell)+\ell \notin S$.

Proof. (i) Since $q \geq 3$ and $\ell<\frac{1}{3}$, we have $\lambda=(1+(q-5) \ell) / 2 \geq(1-2 \ell) / 2>0$. Also, since $q<(q n+2 t) / n=1 / \ell$, we have $\lambda<(1+(1 / \ell-5) \ell) / 2<1-2 \ell$. So, $0<\lambda<1-2 \ell$.

(ii) If $q=3$ (i.e. $\frac{1}{4}<\ell<\frac{1}{3}$ ) then $\lambda=(1-2 \ell) / 2<\ell$. So, by (8) and Lemma 2 , $T_{-}(\lambda)=2 \lambda=1-2 \ell \in A \cap S$, implying that $\lambda \in S$.

If $q \geq 4$ (i.e. $\ell<\frac{1}{4}$ ) then $\ell<(1+(4-5) \ell) / 2 \leq \lambda<1-2 \ell$ (by (i)). By (8) and a simple induction argument, we have $T_{-}^{j}(\lambda)=(1-(q-1) \ell) / 2+(q-3-j) \ell>\ell$ for $0 \leq j \leq q-4$. So $T_{-}^{q-3}(\lambda)=(1-(q-1) \ell) / 2<\ell($ since $(q+1) \ell>1)$ and $T_{-}^{q-2}(\lambda)=1-(q-1) \ell \in A \cap S$ (by Lemma 2), implying that $\lambda \in S$.

(iii) By (i) and $(q+1) \ell>1$, we have $1>\lambda+2 \ell=(1+(q-1) \ell) / 2>1-\ell$. By (7) and (8),

$$
T_{+}(\lambda+2 \ell)=T_{-}(\lambda+2 \ell)=2(\lambda+2 \ell)-1=(q-1) \ell .
$$

Since $(q-1) \ell \in A$ and $(q-1) \ell \notin\{1-j \ell: j=1, \ldots, q-1\}=A \cap S$ (by (6) and Lemma 2), we have $(q-1) \ell \notin S$. By (9) and (14), the Markov chain $\left\{X_{i}\right\}$ defined in (4) satisfies $\mathrm{P}\left(X_{1}=1\right.$ or $\left.(q-1) \ell \mid X_{0}=\lambda+2 \ell\right)=1$, which together with $(q-1) \ell \notin S$ and the definition of $S$, (5), implies that $\lambda+2 \ell \notin S$. The proof is complete.

While Lemma 3 concerns $\ell \in\left(0, \frac{1}{3}\right)$, we partition the interval $\left(\frac{1}{3}, \frac{1}{2}\right)$ into disjoint subintervals,

$$
\left(\frac{1}{3}, \frac{3}{7}\right),\left[\frac{3}{7}, \frac{7}{15}\right),\left[\frac{7}{15}, \frac{15}{31}\right), \ldots,\left[\frac{2^{k-1}-1}{2^{k}-1}, \frac{2^{k}-1}{2^{k+1}-1}\right), \ldots,
$$

so that, for each $\frac{1}{3}<\ell<\frac{1}{2}$, there is a unique $k \geq 2$ such that

$$
\frac{2^{k-1}-1}{2^{k}-1} \leq \ell<\frac{2^{k}-1}{2^{k+1}-1} \text {. }
$$


Lemma 4. Assume that $\ell=n /(q n+2 t)$ satisfies (6) and (15) for some $k \geq 2$ (implying that $q=2)$. Furthermore, assume that $\ell \neq\left(2^{k}-1\right) / 2^{k+1}$. Let $\mu_{j}=(1-\ell) / 2^{j}, j \geq 0$. Then

(i) $0<\mu_{k}<1-2 \ell$;

(ii) $\mu_{k} \in S$;

(iii) $\mu_{k}+2 \ell \notin S$.

Consequently, we have $\mu_{k}+\ell \in(\ell, 1-\ell),\left(\mu_{k}+\ell\right)-\ell \in S$, and $\left(\mu_{k}+\ell\right)+\ell \notin S$.

Proof. (i) By (15), $\ell<\left(2^{k}-1\right) /\left(2^{k+1}-1\right)$, which is equivalent to $1-\ell<2^{k}(1-2 \ell)$. So $0<\mu_{k}=(1-\ell) / 2^{k}<1-2 \ell$.

(ii) Since $\ell>\frac{1}{3}$, we have $\ell>(1-\ell) / 2=\mu_{1}>\mu_{2}>\cdots>\mu_{k}$. By (7), $T_{+}^{k}\left(\mu_{k}\right)=$ $T_{+}^{k-1}\left(T_{+}\left(\mu_{k}\right)\right) \stackrel{3}{=} T_{+}^{k-1}\left(2 \mu_{k}\right)=T_{+}^{k-1}\left(\mu_{k-1}\right)=\cdots=T_{+}\left(\mu_{1}\right)=1-\ell$, implying that $\mu_{k} \in S$.

(iii) By (15), we have, for $0 \leq j \leq k-1$,

$$
\begin{aligned}
\mu_{k-j}+2^{j+1} \ell-2^{j}+1 & =\frac{1}{2^{k-j}}+\left(2^{j+1}-\frac{1}{2^{k-j}}\right) \ell-2^{j}+1 \\
& \geq \frac{1}{2^{k-j}}+\left(2^{j+1}-\frac{1}{2^{k-j}}\right) \frac{2^{k-1}-1}{2^{k}-1}-2^{j}+1 \\
& =1-\frac{2^{j-1}}{2^{k}-1} \\
& >1-\ell .
\end{aligned}
$$

(Note that, by (15), if $k>2, \ell \geq\left(2^{k-1}-1\right) /\left(2^{k}-1\right)>2^{j-1} /\left(2^{k}-1\right)$ for $j \leq k-1$ and, if $k=2, \ell>\frac{1}{3} \geq 2^{j-1} /\left(2^{2}-1\right)$ for $j \leq 1$.) Also, by (i), $1-2 \ell-(1-\ell) / 2^{k}>0$, so, for $0 \leq j \leq k-1$,

$$
\begin{aligned}
\mu_{k-j}+2^{j+1} \ell-2^{j}+1 & =1-2^{j}\left(1-2 \ell-\frac{1-\ell}{2^{k}}\right) \\
& \leq 1-\left(1-2 \ell-\frac{1-\ell}{2^{k}}\right) \\
& <1 .
\end{aligned}
$$

By (7), (8), and (15)-(17), a simple induction argument yields

$$
T_{+}^{j}\left(\mu_{k}+2 \ell\right)=T_{-}^{j}\left(\mu_{k}+2 \ell\right)=\mu_{k-j}+2^{j+1} \ell-2^{j}+1, \quad j=0,1, \ldots, k .
$$

So,

$$
T_{+}^{k}\left(\mu_{k}+2 \ell\right)=T_{-}^{k}\left(\mu_{k}+2 \ell\right)=\mu_{0}+2^{k+1} \ell-2^{k}+1=\left(2^{k+1}-1\right) \ell-\left(2^{k}-2\right) \in A .
$$

But $\left(2^{k+1}-1\right) \ell-\left(2^{k}-2\right) \neq 1-\ell$, since $\ell \neq\left(2^{k}-1\right) / 2^{k+1}$, by assumption. It follows that $\left(2^{k+1}-1\right) \ell-\left(2^{k}-2\right) \notin\{1-\ell\}=\{1-j \ell: j=1, \ldots, q-1\}=A \cap S$ (by Lemma 2), so $\left(2^{k+1}-1\right) \ell-\left(2^{k}-2\right) \notin S$. By (16)-(18), the Markov chain $\left\{X_{i}\right\}$ defined in (4) satisfies

$\mathrm{P}\left(X_{i}>1-\ell, i=1,2, \ldots, k-1\right.$, and $X_{k}=1$ or $\left.\left(2^{k+1}-1\right) \ell-\left(2^{k}-2\right) \mid X_{0}=\mu_{k}+2 \ell\right)=1$.

Since $\left(2^{k+1}-1\right) \ell-\left(2^{k}-2\right) \notin S$, it follows, by the definition of $S,(5)$, that $\mu_{k}+2 \ell \notin S$. The proof is complete. 
Lemma 5. Assume that $\ell=\left(2^{k}-1\right) / 2^{k+1}$ for some $k \geq 2$ (i.e. $n=2^{k}-1, q=2$, and $t=1$ in (6)). Let $v=(1-\ell) / 2^{k-1}-\ell / 2^{2 k-2}$. Then

(i) $0<v<1-2 \ell$;

(ii) $v \in S$;

(iii) $v+2 \ell \notin S$.

Consequently, we have $v+\ell \in(\ell, 1-\ell),(v+\ell)-\ell \in S$, and $(v+\ell)+\ell \notin S$.

Proof. (i) Since $\ell=\left(2^{k}-1\right) / 2^{k+1}$ for $k \geq 2$, we have

$$
0<v=\frac{1-\ell}{2^{k-1}}-\frac{\ell}{2^{2 k-2}}=\frac{1}{2^{k}}-\left(\frac{1}{2^{2 k}}-\frac{1}{2^{3 k-1}}\right)<\frac{1}{2^{k}}=1-2 \ell .
$$

(ii) For $0 \leq j \leq k-2$, we have $0<2^{j} v<(1-\ell) / 2^{k-j-1} \leq(1-\ell) / 2<\ell$, and

$$
\ell=\frac{2^{k}-1}{2^{k+1}}<\frac{2^{2 k-1}-2^{k-1}+1}{2^{2 k}}=2^{k-1} v=1-\ell-\frac{\ell}{2^{k-1}}<1-\ell .
$$

By (7),

$$
\begin{aligned}
T_{+}^{2 k-1}(v) & =T_{+}^{k}\left(T_{+}^{k-1}(v)\right) \\
& =T_{+}^{k}\left(2^{k-1} v\right) \\
& =T_{+}^{k}\left(1-\ell-\frac{\ell}{2^{k-1}}\right) \\
& =T_{+}^{k-1}\left(1-\frac{\ell}{2^{k-1}}\right) \\
& =T_{+}^{k-2}\left(1-\frac{\ell}{2^{k-2}}\right) \\
& =\cdots \\
& =1-\ell
\end{aligned}
$$

implying that $v \in S$.

(iii) Note that $v+2 \ell=1-\left(2^{-2 k}-2^{-3 k+1}\right)$. Since we have, for $0 \leq j \leq 2 k-2$,

$$
\begin{aligned}
1 & >1-\left(\frac{1}{2^{2 k-j}}-\frac{1}{2^{3 k-j-1}}\right) \\
& =1-2^{j}\left(\frac{1}{2^{2 k}}-\frac{1}{2^{3 k-1}}\right) \\
& \geq 1-2^{2 k-2}\left(\frac{1}{2^{2 k}}-\frac{1}{2^{3 k-1}}\right) \\
& =1-\frac{1}{4}+\frac{1}{2^{k+1}} \\
& >1-\ell,
\end{aligned}
$$

it follows, by induction and (7)-(8), that, for $0 \leq j \leq 2 k-2$,

$$
T_{+}^{j}(\nu+2 \ell)=T_{-}^{j}(\nu+2 \ell)=1-\left(\frac{1}{2^{2 k-j}}-\frac{1}{2^{3 k-j-1}}\right)>1-\ell .
$$


In particular,

$$
T_{+}^{2 k-2}(v+2 \ell)=T_{-}^{2 k-2}(\nu+2 \ell)=\frac{3}{4}+\frac{1}{2^{k+1}} \in A=\left\{\frac{j}{2^{k+1}}: j=1, \ldots, 2^{k+1}-1\right\} .
$$

Since $1-\ell<\frac{3}{4}+1 / 2^{k+1}<1$, we have $\frac{3}{4}+1 / 2^{k+1} \notin\{1-\ell\}=\{1-j \ell: j=1, \ldots, q-1\}=$ $A \cap S$ (by Lemma 2), implying that $\frac{3}{4}+1 / 2^{k+1} \notin S$. By (19), the Markov chain $\left\{X_{i}\right\}$ defined in (4) satisfies

$$
\mathrm{P}\left(X_{i}>1-\ell, i=1,2, \ldots, 2 k-2, \text { and } X_{2 k-2}=1 \text { or } \frac{3}{4}+\frac{1}{2^{k+1}} \mid X_{0}=v+2 \ell\right)=1 .
$$

Since $\frac{3}{4}+1 / 2^{k+1} \notin S$, it follows, by the definition of $S$, (5), that $v+2 \ell \notin S$, completing the proof.

\section{The case in which $\ell=n /(2 n+1)$ under condition (3)}

In this section we assume that $\ell$ is of the form $n /(2 n+1), n \geq 2$. Define a function $T:[0,1] \rightarrow[0,1]$ by $T(f)=2 f-\lfloor 2 f\rfloor$ for $0 \leq f<1$ and $T(1)=1$, where $\lfloor x\rfloor$ denotes the largest integer less than or equal to $x$. Note that $T(f)=T_{+, \ell}(f)=T_{-, \ell}(f)$ for $f \in[0, \ell] \cup[1-\ell, 1]$. It follows by induction that

$$
T^{i}(f)=2^{i} f-\left\lfloor 2^{i} f\right\rfloor, \quad 0 \leq f<1, i=1,2, \ldots
$$

Letting (cf. (10))

$$
A=A_{\ell}=\left\{\frac{j}{2 n+1}: j=1,2, \ldots, 2 n\right\},
$$

we have the following simple but useful results on the Markov chain $\left\{X_{i}\right\}$ defined in (4):

$$
\mathrm{P}\left(X_{i+1}=T\left(X_{i}\right) \mid X_{i}=x\right)>0, \quad \mathrm{P}\left(X_{i+1}=T\left(X_{i}\right) \text { or } 0 \text { or } 1 \mid X_{i}=x\right)=1,
$$

for all $x \in A$ and $i \geq 0$. Since $f \in A$ implies that $T(f) \in A$, it follows from (21) that

$$
\mathrm{P}\left(X_{i}=T^{i}\left(X_{0}\right) \mid X_{0}=x\right)>0, \quad \mathrm{P}\left(X_{i}=T^{i}\left(X_{0}\right) \text { or } 0 \text { or } 1 \mid X_{0}=x\right)=1,
$$

for all $x \in A$ and $i \geq 1$. Letting $\alpha$ be the smallest positive integer satisfying $2^{\alpha} \equiv 1$ $(\bmod 2 n+1)$, we have, by $(20)$, for $m=1, \ldots, 2 n$,

$$
T^{\alpha}\left(\frac{m}{2 n+1}\right)=\frac{2^{\alpha} m}{2 n+1}-\left\lfloor\frac{2^{\alpha} m}{2 n+1}\right\rfloor=\frac{m}{2 n+1}-\left\lfloor\frac{m}{2 n+1}\right\rfloor=\frac{m}{2 n+1} .
$$

Lemma 6. For $\ell=n /(2 n+1), n \geq 2$, we have

$$
A \cap S=\left\{T^{i}\left(\frac{1}{2 n+1}\right): i=0,1, \ldots, \alpha-1\right\} .
$$

Proof. We first claim that

$$
x=n+1 \text { is the only integer in }\{1, \ldots, 2 n\} \text { that satisfies } T\left(\frac{x}{2 n+1}\right)=\frac{1}{2 n+1} .
$$


To see this, note that if $\lfloor 2 x /(2 n+1)\rfloor=0$ then

$$
T\left(\frac{x}{2 n+1}\right)=\frac{2 x}{2 n+1}-\left\lfloor\frac{2 x}{2 n+1}\right\rfloor=\frac{2 x}{2 n+1} \neq \frac{1}{2 n+1},
$$

implying that $x$ must satisfy $\lfloor 2 x /(2 n+1)\rfloor=1$ in order to solve $T(x /(2 n+1))=1 /(2 n+1)$. The claim then follows readily. Since $T^{\alpha}(1 /(2 n+1))=1 /(2 n+1)$ by $(23)$ and since $T^{\alpha-1}(1 /(2 n+1)) \in A$, it follows from (24) that $T^{\alpha-1}(1 /(2 n+1))=(n+1) /(2 n+1)=1-\ell$. So $\left\{T^{i}(1 /(2 n+1)): i=0,1, \ldots, \alpha-1\right\} \subset A \cap S$ by (22) and the definition of $S$, (5).

To show that $A \cap S \subset\left\{T^{i}(1 /(2 n+1)): i=0,1, \ldots, \alpha-1\right\}$, suppose that $m /(2 n+1) \in A \cap S$ for some $m \in\{1, \ldots, 2 n\}$. By (5), (22), and (23), there exists an integer $0 \leq j \leq \alpha-1$ such that $T^{j}(m /(2 n+1))=1-\ell$. Then $T^{j+1}(m /(2 n+1))=T(1-\ell)=1 /(2 n+1)$. By $(23)$, we have

$$
\begin{aligned}
\frac{m}{2 n+1} & =T^{\alpha}\left(\frac{m}{2 n+1}\right) \\
& =T^{\alpha-j-1}\left(T^{j+1}\left(\frac{m}{2 n+1}\right)\right) \\
& =T^{\alpha-j-1}\left(\frac{1}{2 n+1}\right) \in\left\{T^{i}\left(\frac{1}{2 n+1}\right): i=0,1, \ldots, \alpha-1\right\} .
\end{aligned}
$$

The proof is complete.

Lemma 7. Assume that $\ell=n /(2 n+1)$ satisfies (3), i.e. $\alpha<2 n$. Then $A \backslash S \neq \varnothing$ and

$$
A \backslash S \subset\left\{\frac{m}{2 n+1} \in A: m \neq 2^{j} \text { for any integer } j \geq 0\right\} .
$$

Proof. Denoting by $|R|$ the cardinality of a set $R$, we have, by Lemma 6 and (3), $|A \cap S|=$ $\alpha<2 n=|A|$; so $A \backslash S \neq \varnothing$. It remains to show that if $m /(2 n+1) \in A \backslash S$ then $m \neq 2^{j}$ for any integer $j \geq 0$. This follows from the fact that, for any integer $j \geq 0$ with $2^{j} \leq 2 n$, we have, by (20) and Lemma 6,

$$
\frac{2^{j}}{2 n+1}=\frac{2^{j}}{2 n+1}-\left\lfloor\frac{2^{j}}{2 n+1}\right\rfloor=T^{j}\left(\frac{1}{2 n+1}\right) \in A \cap S .
$$

The proof is complete.

Lemma 8. For $\ell=n /(2 n+1), n \geq 2$, assume that $1-1 /(2 n+1) \in A \cap S$. Then, for $m \in\{1,2, \ldots, 2 n\}$, we have $m /(2 n+1) \in A \cap S$ if and only if $1-m /(2 n+1) \in A \cap S$.

Proof. For integers $j \geq 0$ and $1 \leq m \leq 2 n$, we have, by (20),

$$
\begin{aligned}
T^{j}\left(1-\frac{m}{2 n+1}\right) & =2^{j}-\frac{2^{j} m}{2 n+1}-\left\lfloor 2^{j}-\frac{2^{j} m}{2 n+1}\right\rfloor \\
& =-\left(\left\lfloor\frac{2^{j} m}{2 n+1}\right\rfloor+\left\lfloor-\frac{2^{j} m}{2 n+1}\right\rfloor\right)-\left(\frac{2^{j} m}{2 n+1}-\left\lfloor\frac{2^{j} m}{2 n+1}\right\rfloor\right) \\
& =1-T^{j}\left(\frac{m}{2 n+1}\right),
\end{aligned}
$$

since $2^{j} m \not \equiv 0(\bmod 2 n+1)$ and since $\lfloor x\rfloor+\lfloor-x\rfloor=-1$ for any positive noninteger $x$. 
It suffices to show that $m /(2 n+1) \in A \cap S$ implies that $1-m /(2 n+1) \in A \cap S$. Suppose that $m /(2 n+1) \in A \cap S$. Then, by (5) and (22), there exists an integer $j \geq 0$ such that $T^{j}(m /(2 n+1))=1-\ell$. It follows from $(25)$ that

$$
T^{j+1}\left(1-\frac{m}{2 n+1}\right)=1-T^{j+1}\left(\frac{m}{2 n+1}\right)=1-T(1-\ell)=1-\frac{1}{2 n+1} .
$$

Since $1-1 /(2 n+1) \in A \cap S$ by assumption, we have $1-m /(2 n+1) \in A \cap S$. The proof is complete.

We are now ready to state and prove the following main result of this section, which together with Lemma 1 implies that (1) holds for $\ell=n /(2 n+1)$ satisfying (3).

Theorem 2. Assume that $\ell=n /(2 n+1)$ satisfies (3). Then there exists an $f \in(\ell, 1-\ell)$ such that $f-\ell \in S$ and $f+\ell \notin S$.

Proof. If $1-1 /(2 n+1) \notin A \cap S$, we have, with $f=\frac{1}{2} \in(\ell, 1-\ell)$,

$$
T(f-\ell)=2 f-2 \ell=\frac{1}{2 n+1} \in A \cap S \quad \text { (by Lemma 6) }
$$

and

$$
T(f+\ell)=2(f+\ell)-1=1-\frac{1}{2 n+1} \notin A \cap S .
$$

Since $0<f-\ell<\ell$ and $1-\ell<f+\ell<1$, the Markov chain $\left\{X_{i}\right\}$ defined in (4) satisfies

$\mathrm{P}\left(X_{1}=T(f-\ell)\right.$ or $\left.0 \mid X_{0}=f-\ell\right)=1, \quad \mathrm{P}\left(X_{1}=T(f+\ell)\right.$ or $\left.1 \mid X_{0}=f+\ell\right)=1$.

It follows from (26)-(28) that $f-\ell \in S$ and $f+\ell \notin S$.

We now assume that $1-1 /(2 n+1) \in A \cap S$. By Lemma 7 , let $a /(2 n+1)$ be the smallest element of $A \backslash S \neq \varnothing$, so that

$$
\frac{a}{2 n+1} \in A \backslash S \text { and } \quad 2^{k-1}<a<2^{k} \quad \text { for some integer } k \geq 2 .
$$

By Lemma $8, a /(2 n+1) \notin A \cap S$ implies that

$$
1-\frac{a}{2 n+1} \notin A \cap S
$$

Since $a /(2 n+1)$ is the smallest element of $A \backslash S$, it follows that $a /(2 n+1) \leq 1-a /(2 n+1)$; so $a \leq n$. But $a=n$ would lead to $1-a /(2 n+1)=1-\ell \in A \cap S$, contradicting (30). Thus, $a<n$.

Letting $b=2^{k}-a$, we have, by (29),

$$
1 \leq b<2^{k}-2^{k-1}=2^{k-1}<a<n .
$$

Since $a /(2 n+1)$ is the smallest element of $A \backslash S, 1 \leq b<a$ implies that

$$
\frac{b}{2 n+1} \in A \cap S \text {. }
$$


Let $f=1-\ell-a /\left(2^{k}(2 n+1)\right)$. By (29), it is easily checked that $\ell<f<1-\ell$. Also, by (20) and (31), for $i=1, \ldots, k$,

$$
\begin{aligned}
T^{i}(f-\ell) & =T^{i}\left(\frac{b}{2^{k}(2 n+1)}\right) \\
& =\frac{b}{2^{k-i}(2 n+1)}-\left\lfloor\frac{b}{2^{k-i}(2 n+1)}\right\rfloor \\
& =\frac{b}{2^{k-i}(2 n+1)} \\
& <\frac{\ell}{2^{k-i}} \\
& \leq \ell, \\
T^{i}(f+\ell) & =T^{i}\left(1-\frac{a}{2^{k}(2 n+1)}\right) \\
& =2^{i}-\frac{a}{2^{k-i}(2 n+1)}-\left\lfloor 2^{i}-\frac{a}{2^{k-i}(2 n+1)}\right\rfloor \\
& =1-\frac{a}{2^{k-i}(2 n+1)} \\
& >1-\frac{n}{2^{k-i}(2 n+1)} \\
& =1-\frac{\ell}{2^{k-i}} \\
& \geq 1-\ell .
\end{aligned}
$$

It follows that

$$
T^{i}(f-\ell)<\ell \text { and } T^{i}(f+\ell)>1-\ell \text { for } i=1, \ldots, k
$$

and, by (30) and (32), that

$$
T^{k}(f-\ell)=\frac{b}{2 n+1} \in A \cap S \quad \text { and } \quad T^{k}(f+\ell)=1-\frac{a}{2 n+1} \notin A \cap S .
$$

Recalling that $T=T_{+}=T_{-}$on $[0, \ell] \cup[1-\ell, 1]$, for the Markov chain $\left\{X_{i}\right\}$ defined in (4), we have, by (33), for $i=1, \ldots, k$,

$\mathrm{P}\left(X_{i}=T^{i}(f-\ell)\right.$ or $\left.0 \mid X_{0}=f-\ell\right)=1, \quad \mathrm{P}\left(X_{i}=T^{i}(f+\ell)\right.$ or $\left.1 \mid X_{0}=f+\ell\right)=1$, which together with (34) and the definition of $S$, (5), implies that $f-\ell \in S$ and $f+\ell \notin S$. The proof is complete.

\section{Further remarks on the case in which $\ell=n /(2 n+1)$}

Theorem 2 in Section 4 requires that $\alpha<2 n$, where $\alpha$ is the smallest positive integer satisfying $2^{\alpha} \equiv 1(\bmod 2 n+1)$. As noted in Section 1 , it follows from Euler's theorem that $\alpha \leq 2 n$ and that $\alpha<2 n$ if $2 n+1$ is not a prime. In view of Theorem 2 , the case in which $\alpha=2 n$ (implying that $2 n+1$ is a prime) remains open. The following result shows that this case cannot be settled by Schweinsberg's [5] method. 
Proposition 1. Let $\ell=n /(2 n+1)$ for some $n \geq 2$. Assume that $2 n+1$ is a prime and $\alpha=2 n$. Then there exists no $f \in(\ell, 1-\ell)$ such that $f-\ell \in S$ and $f+\ell \notin S$.

Proof. It suffices to show that if $f$ satisfies $f \in(\ell, 1-\ell)$ and $f-\ell \in S$, then $f+\ell \in S$. Let $\left\{X_{i}\right\}$ be the Markov chain defined in (4). By the definition of $S$, (5), $f-\ell \in S$ implies that there exist an integer $I \geq 1$ and nonabsorbing states $x_{1}, \ldots, x_{I} \in(0,1)$ such that $x_{I}=1-\ell$ and $\mathrm{P}\left(X_{i}=x_{i}, i=1, \ldots, I \mid X_{0}=f-\ell\right)>0$. With $x_{0}:=f-\ell$, it is readily seen that if $x_{i} \in(0, \ell] \cup[1-\ell, 1)(0 \leq i \leq I-1)$ then $x_{i+1}=2 x_{i}-\left\lfloor 2 x_{i}\right\rfloor\left(=T\left(x_{i}\right)=T_{+}\left(x_{i}\right)=T_{-}\left(x_{i}\right)\right)$; and if $x_{i} \in(\ell, 1-\ell)$ then $x_{i+1}=x_{i}+\ell\left(=T_{+}\left(x_{i}\right)\right)$ or $x_{i}-\ell\left(=T_{-}\left(x_{i}\right)\right)$.

Let $V=\left\{x \in(0,1): \mathrm{P}\left(X_{j}=x \mid X_{0}=f+\ell\right)>0\right.$ for some $\left.j \geq 0\right\}$ be the set of nonabsorbing states that the Markov chain could visit starting from $f+\ell$. We claim that if there exists a $y \in V$ such that $\left|y-x_{i}\right| \in A_{0}:=\{m /(2 n+1): m=0,1, \ldots, 2 n\}$ then there exists a $y^{\prime} \in V$ such that $\left|y^{\prime}-x_{i+1}\right| \in A_{0}$. To see this, note that if $\left|y-x_{i}\right|=0$ then the claim holds trivially by taking $y^{\prime}=x_{i+1}$ (which is necessarily in $V$ since $x_{i}=y \in V$ ). We now assume that $0<\left|y-x_{i}\right| \in A_{0}$, in which case at most one of $y$ and $x_{i}$ can be in $(\ell, 1-\ell)$, since $\left|y-x_{i}\right| \geq 1 /(2 n+1)=(1-\ell)-\ell$. So it suffices to consider the following cases.

(i) $y, x_{i} \in(0, \ell] \cup[1-\ell, 1)$. Letting $y^{\prime}=T(y) \in V$ (since $\left.y \in V\right)$, we have $\left|y^{\prime}-x_{i+1}\right|=$ $\left|T(y)-T\left(x_{i}\right)\right| \in A_{0}\left(\right.$ since $\left.\left|y-x_{i}\right| \in A_{0}\right)$.

(ii) $y \in(0, \ell] \cup[1-\ell, 1)$ and $x_{i} \in(\ell, 1-\ell)$. Letting $y^{\prime}=y \in V$, we have $\left|y^{\prime}-x_{i+1}\right|=$ $\left|y-x_{i+1}\right|=\left|y-x_{i}-\ell\right|$ or $\left|y-x_{i}+\ell\right|$ (depending on whether $x_{i+1}=x_{i}+\ell$ or $\left.x_{i+1}=x_{i}-\ell\right)$, implying that $\left|y^{\prime}-x_{i+1}\right| \in A_{0}$.

(iii) $y \in(\ell, 1-\ell)$ and $x_{i} \in(0, \ell] \cup[1-\ell, 1)$. Letting $y^{\prime}=T(y+\ell) \in V$, we have $\left|y^{\prime}-x_{i+1}\right|=\left|T(y+\ell)-T\left(x_{i}\right)\right| \in A_{0}$, since $\left|y-x_{i}\right| \in A_{0}$ implies that $\left|y+\ell-x_{i}\right| \in A_{0}$.

This proves the claim.

Since $f+\ell \in V$ and $\left|(f+\ell)-x_{0}\right|=2 \ell \in A_{0}$, it follows from the claim that there exist $y_{1}, \ldots, y_{I} \in V$ such that $\left|y_{i}-x_{i}\right| \in A_{0}, i=1, \ldots, I$. In particular, we have $y_{I} \in V$ and $\left|y_{I}-(1-\ell)\right|=\left|y_{I}-x_{I}\right| \in A_{0}$, implying that $y_{I} \in A:=\{m /(2 n+1): m=1, \ldots, 2 n\}$. By Lemma 6 and $\alpha=2 n$, we have $|A \cap S|=\alpha=2 n=|A|$, i.e. $A=A \cap S \subset S$. So $y_{I} \in A \subset S$, which together with $y_{I} \in V$ implies that $f+\ell \in S$. The proof is complete.

While Proposition 1 shows that Schweinsberg's [5] method cannot apply to the case in which $\ell=n /(2 n+1)$ with $n \geq 2$ and $\alpha=2 n$, we now argue that it is possible to establish the validity of (1) for such $\ell$ with the help of symbolic-computation software.

Fix an $\ell=n /(2 n+1)$ with $n \geq 2$ and $\alpha=2 n$. Let

$$
\begin{aligned}
G_{0} & =G_{0, \ell}=\left\{\frac{i}{2 n+1}: i=1,2, \ldots, 2 n\right\}, \\
G_{j} & =G_{j, \ell}=\left\{\frac{i}{2^{j}(2 n+1)}: i=1,3,5, \ldots, 2^{j}(2 n+1)-1\right\}, \quad j=1,2, \ldots
\end{aligned}
$$

Clearly, $G_{0}, G_{1}, G_{2}, \ldots$ are disjoint and

$$
\bigcup_{j=0}^{m} G_{j}=\left\{\frac{i}{2^{m}(2 n+1)}: i=1,2, \ldots, 2^{m}(2 n+1)-1\right\}, \quad m=0,1, \ldots
$$


As a function of $w, Q_{\ell, w}(f)$ can be found easily for $f \in \bigcup_{j=0}^{\infty} G_{j}$ as follows. We first determine $Q_{\ell, w}(f), f \in G_{0}$, by solving the following set of $2 n$ linear equations:

$$
\begin{aligned}
Q_{\ell, w}\left(\frac{i}{2 n+1}\right) & =w Q_{\ell, w}\left(\frac{2 i}{2 n+1}\right), \quad i=1, \ldots, n \\
Q_{\ell, w}\left(\frac{i}{2 n+1}\right) & =w+\bar{w} Q_{\ell, w}\left(\frac{2 i-2 n-1}{2 n+1}\right), \quad i=n+1, \ldots, 2 n,
\end{aligned}
$$

where $\bar{w}=1-w$, or, equivalently,

$$
\begin{aligned}
Q_{\ell, w} & \left.T^{i}\left(\frac{1}{2 n+1}\right)\right) \\
= & \begin{cases}w Q_{\ell, w}\left(T^{i+1}\left(\frac{1}{2 n+1}\right)\right) & \text { if } T^{i}\left(\frac{1}{2 n+1}\right) \leq \frac{n}{2 n+1}=\ell, \\
w+\bar{w} Q_{\ell, w}\left(T^{i+1}\left(\frac{1}{2 n+1}\right)\right) & \text { if } T^{i}\left(\frac{1}{2 n+1}\right) \geq \frac{n+1}{2 n+1}=1-\ell,\end{cases}
\end{aligned}
$$

since $\left\{T^{i}(1 /(2 n+1)): i=1, \ldots, 2 n\right\}=\{i /(2 n+1): i=1, \ldots, 2 n\}($ by $\alpha=2 n)$. Letting $x=Q_{\ell, w}(1 /(2 n+1))$, we have $Q_{\ell, w}(T(1 /(2 n+1)))=x / w, Q_{\ell, w}\left(T^{2}(1 /(2 n+1))\right)=x / w^{2}$ (since $T(1 /(2 n+1)) \leq n /(2 n+1))$, and $Q_{\ell, w}\left(T^{3}(1 /(2 n+1))\right)=x / w^{3}$ or $x /\left(w^{2} \bar{w}\right)-w / \bar{w}$ according to whether $T^{2}(1 /(2 n+1)) \leq n /(2 n+1)$ or $T^{2}(1 /(2 n+1)) \geq(n+1) /(2 n+1)$. More generally, let $1<I(1)<I(2)<\cdots<I(n-1)<I(n)=2 n-1$ be such that $T^{I(k)}(1 /(2 n+1)) \geq(n+1) /(2 n+1), k=1, \ldots, n$, and $T^{i}(1 /(2 n+1)) \leq n /(2 n+1)$ for $i \in\{1,2, \ldots, 2 n\} \backslash\{I(1), \ldots, I(n)\}$. (Note that $T^{2 n-1}(1 /(2 n+1))=(n+1) /(2 n+1)$ since $T((n+1) /(2 n+1))=1 /(2 n+1)$.) Letting $I(0):=0$ and $I(n+1):=2 n$, we have, for $I(k)<i \leq I(k+1), k=0,1, \ldots, n$,

$$
Q_{\ell, w}\left(T^{i}\left(\frac{1}{2 n+1}\right)\right)=\frac{x}{w^{i-k} \bar{w}^{k}}-\sum_{j=1}^{k} \frac{w}{w^{i-I(j)-k+j-1} \bar{w}^{k-j+1}} .
$$

In particular,

$$
x=Q_{\ell, w}\left(T^{2 n}\left(\frac{1}{2 n+1}\right)\right)=\frac{x}{w^{n} \bar{w}^{n}}-\sum_{j=1}^{n} \frac{w}{w^{n-I(j)+j-1} \bar{w}^{n-j+1}},
$$

from which $x=Q_{\ell, w}(1 /(2 n+1))$ and $Q_{\ell, w}(i /(2 n+1)), i=2, \ldots, 2 n$, are obtained.

Now suppose that $Q_{\ell, w}(f), f \in G_{j}$, have been obtained. To find $Q_{\ell, w}(f), f \in G_{j+1}$, we first compute $Q_{\ell, w}(f)$ for $f \in G_{j+1} \cap\{(0, \ell] \cup[1-\ell, 1)\}$ by

$$
\begin{aligned}
& Q_{\ell, w}\left(\frac{i}{2^{j+1}(2 n+1)}\right) \\
& \quad= \begin{cases}w Q_{\ell, w}\left(\frac{i}{2^{j}(2 n+1)}\right) & \text { if } i=1,3,5, \ldots, 2^{j+1} n-1, \\
w+\bar{w} Q_{\ell, w}\left(\frac{i-2^{j}(2 n+1)}{2^{j}(2 n+1)}\right) & \text { if } i=2^{j+1}(n+1)+1, \ldots, 2^{j+1}(2 n+1)-1 .\end{cases}
\end{aligned}
$$


TABLE 1.

\begin{tabular}{cccc}
\hline$\ell$ & $f$ & $s$ & $Q_{\ell, w}(f)-\left(w Q_{\ell, w}(f+s)+\bar{w} Q_{\ell, w}(f-s)\right)$ \\
\hline$\frac{2}{5}$ & $\frac{303}{640}$ & $\frac{2}{5}-\frac{1}{640}$ & $-\frac{w^{5} \bar{w}^{7}(1-2 w)}{1-w^{2} \bar{w}^{2}}$ \\
$\frac{5}{11}$ & $\frac{22431}{45056}$ & $\frac{5}{11}-\frac{1}{45056}$ & $-\frac{w^{6} \bar{w}^{9}(1-2 w)\left[w^{6}\left(5+3 \bar{w}+\bar{w}^{2}\right)+(1-2 w)\left(1+w \bar{w}+3 w^{3}+3 w^{4}\right)\right]}{1-w^{5} \bar{w}^{5}}$ \\
$\frac{6}{13}$ & $\frac{26295}{53248}$ & $\frac{6}{13}-\frac{1}{53248}$ & $-\frac{w^{9} \bar{w}^{6}(1-2 w) P_{1}(w)}{1-w^{6} \bar{w}^{6}}$ \\
$\frac{9}{19}$ & $\frac{19503}{38912}$ & $\frac{9}{19}-\frac{1}{38912}$ & $-\frac{w^{6} \bar{w}^{7}(1-2 w) P_{2}(w)}{1-w^{9} \bar{w}^{9}}$ \\
$\frac{14}{29}$ & $\frac{29105}{59392}$ & $\frac{14}{29}-\frac{1}{59392}$ & $-\frac{w^{9} \bar{w}^{10}(1-2 w)\left(1+w \bar{w}^{2}\right)\left(1+w^{2} \bar{w}\right)^{2}\left(1-w^{2} \bar{w}+w^{5} \bar{w}^{2}\right)}{1-w^{14} \bar{w}^{14}}$ \\
\hline
\end{tabular}

We then compute $Q_{\ell, w}(f)$ for $f \in G_{j+1} \cap(\ell, 1-\ell)$ by

$$
Q_{\ell, w}\left(\frac{i}{2^{j+1}(2 n+1)}\right)=w Q\left(\frac{i+2^{j+1} n}{2^{j+1}(2 n+1)}\right)+\bar{w} Q\left(\frac{i-2^{j+1} n}{2^{j+1}(2 n+1)}\right),
$$

$i=2^{j+1} n+1, \ldots, 2^{j+1}(n+1)-1$. (Since $\ell>\frac{1}{3}, \ell<f<1-\ell$ implies that $f+\ell>1-\ell$ and $f-\ell<\ell$.)

While the above algorithm shows that $Q_{\ell, w}(f), f \in \bigcup_{j=0}^{\infty} G_{j}$, can be found in a simple recursive fashion, we have used MAPLE ${ }^{\circledR}$ to carry out extensive symbolic computations for $\ell=\frac{2}{5}, \frac{5}{11}, \frac{6}{13}, \frac{9}{19}$, and $\frac{14}{29}$, the first five values of $\ell=n /(2 n+1)$ with $n \geq 2$ and $\alpha=2 n$. By an extensive search, we found a pair of $(f, s)$ with $s<\ell<f<1-\ell$ for each of these five values of $\ell$ such that

$$
\Delta_{\ell, w}(f, s):=Q_{\ell, w}(f)-\left(w Q_{\ell, w}(f+s)+\bar{w} Q_{\ell, w}(f-s)\right)<0 \quad \text { for all } 0<w<\frac{1}{2} .
$$

Note that $U_{\ell, w}(f) \geq w Q_{\ell, w}(f+s)+\bar{w} Q_{\ell, w}(f-s)=Q_{\ell, w}(f)-\Delta_{\ell, w}(f, s)$. Table 1 presents $f, s$, and $\Delta_{\ell, w}(f, s)$ for $\ell=\frac{2}{5}, \frac{5}{11}, \frac{6}{13}, \frac{9}{19}$, and $\frac{14}{29}$, in which the polynomials $P_{1}(w)$ and $P_{2}(w)$ are given as follows, expressed in such a way that they can be readily seen to be strictly positive for $0<w<\frac{1}{2}$ :

$$
\begin{aligned}
P_{1}(w)= & w^{8} \bar{w}+(1-2 w)\left[w \bar{w}+9 w^{3} \bar{w}+\left(1-4 w^{2}\right)+w^{5}\left(5-w^{2}\right)\right], \\
P_{2}(w)= & w^{4} \bar{w}^{2}\left[\left(1-2 w^{3}\right)+w^{4}(3-5 w)+w^{6}(19-33 w)+w^{8}(13+17 \bar{w})+w^{10}(5+\bar{w})\right] \\
& +(1-2 w)\left[1+w^{2} \bar{w}^{2}(1+w)+w^{6} \bar{w}^{2}(2+\bar{w})\right] .
\end{aligned}
$$

\section{Acknowledgements}

The first author gratefully acknowledges support from the National Science Council of Taiwan under grant NSC 096-2811-M-001-065. The third author gratefully acknowledges support from the National Science Council of Taiwan under grant NSC 096-2118-M-018-002. The fourth author gratefully acknowledges support from the National Science Council of Taiwan under grants NSC 094-2118-M-001-019 and NSC 096-2628-M-001-023. 


\section{References}

[1] BAKer, A. (1984). A Concise Introduction to the Theory of Numbers. Cambridge University Press.

[2] Dubins, L. E. And Savage, L. J. (1965). How to Gamble if You Must. Inequalities for Stochastic Processes. McGraw-Hill, New York

[3] Dubins, L. E. and Savage, L. J. (1976). Inequalities for Stochastic Processes. How to Gamble if You Must. Corrected republication of the 1965 edition. Dover, New York.

[4] Heath, D. C., Pruitt, W. E. And Sudderth, W. D. (1972). Subfair red-and-black with a limit. Proc. Amer. Math. Soc. 35, 555-560.

[5] Schweinsberg, J. (2005). Improving on bold play when the gambler is restricted. J. Appl. Prob. 42, 321-333.

[6] Wilkins, J. E. (1972). The bold strategy in presence of house limit. Proc. Amer. Math. Soc. 32, 567-570.

[7] YaO, Y.-C. (2007). On optimality of bold play for discounted Dubins-Savage gambling problems with limited playing times. J. Appl. Prob. 44, 212-225. 